\title{
A METHOD TO FIND THE MEMBERSHIP FUNCTIONS OF MAXIMUM AND MINIMUM OF FUZZY NUMBERS
}

\author{
Shapla Shirin \\ Department of Mathematics \\ University of Dhaka, Dhaka-1000, Bangladesh \\ Email: shapla@univdhaka.edu
}

Received 23.03.09 Accepted 02.01.11

\begin{abstract}
In this paper, a new approach for computation of membership functions of the maximum and minimum of more than two upper semi-continuous fuzzy numbers has been introduced. This method is also applicable for piece-wise continuous fuzzy numbers or the fuzzy numbers which are only continuous from right or only continuous from left. The core of fuzzy numbers should have a singleton set.

Keywords: Continuous fuzzy number, piece-wise continuous fuzzy number, Maximum (MAX) and Minimum (MIN) of fuzzy number, core, $\alpha$-cut, bounded increasing and bounded decreasing functions, membership function.
\end{abstract}

\section{Introduction}

Chiu and Wang [1] introduced a method to implement the operations MIN and MAX for two continuous fuzzy numbers. This is a step-by-step method and takes more time. HONG and KIM [5] proposed a different method to compute $\alpha$-cuts the maximum and the minimum of more than two continuous and non-continuous fuzzy numbers via $\alpha$-cut presentation.

In this paper, a new method is investigated which can compute the membership functions of the maximum and the minimum of more than two fuzzy numbers easily and quickly. The resulting membership function may be the previous one of the fuzzy numbers or the combination of their different parts. For this, we need to find the intersecting points of the membership functions of fuzzy numbers. The notion of this method has not been focused by anybody till now.

\section{Preliminaries}

L. A. Zadeh [7 ] first introduced the concept of fuzzy sets. A fuzzy number $\mu$ is a fuzzy set on the real line $\mathrm{P}$ which is defined as follows [6]: 
Definition 2.1: A fuzzy set $\mu: \mathrm{P} \rightarrow I=[0,1]$ is said to be a fuzzy number if it possesses the following properties:

(a) $\mu$ is a normal fuzzy set;

(b) for every $\alpha \in(0,1]$, the $\alpha$-cut of $\mu,{ }^{\alpha} \mu$, is a closed interval denoted by $[\mu(\alpha), \bar{\mu}(\alpha)]$

(c) the support of $\mu,{ }^{0+} \mu$, is bounded.

We shall denote the set of all fuzzy numbers by $\phi v(\mathrm{P})$.

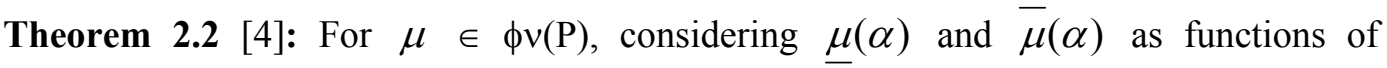
$\alpha \in[0,1]:$

(a) $\mu(\alpha)$ is a bounded increasing function on $[0,1]$.

(b) $\bar{\mu}(\alpha)$ is a bounded decreasing function on $[0,1]$.

(c) $\mu(\alpha) \leq \bar{\mu}(\alpha)$.

(d) $\underline{\mu}(\alpha)$ and $\bar{\mu}(\alpha)$ are left continuous on $[0,1]$ and right continuous at 0 .

(e) If $\underline{v}(\alpha)$ and $\bar{v}(\alpha)$ satisfy (a) (d), then there exists a unique $v \in \phi v(\mathrm{P})$ such that ${ }^{\alpha} v=[\underline{v}(\alpha), \bar{v}(\alpha)]$.

Other contributors $[1,5,6]$ of the operations MAX and MIN used the above definition 2.1 and the theorem 2.2. In this paper, we use the following definition of a fuzzy number.

Definition $2.2[2,3]$ : A fuzzy set $\mu$ is called a fuzzy number if and only if for real numbers ${ }^{\alpha} a,{ }^{\alpha} c,{ }^{\alpha} b$ the following properties are satisfied:

(a) $\mu(x)=1$ for $x={ }^{\alpha} c$;

(b) for every $\alpha \in I_{0}=(0,1]$,

${ }^{\alpha} \mu=[\underline{\mu}(\alpha), \bar{\mu}(\alpha)]=\left[{ }^{\alpha} a+\left({ }^{\alpha} c-{ }^{\alpha} a\right) \alpha,{ }^{\alpha} b-\left({ }^{\alpha} b-{ }^{\alpha} c\right) \alpha\right]$.

(c) ${ }^{0+} \mu=\left({ }^{\alpha} a,{ }^{\alpha} b\right)$. 
Such a fuzzy number will be denoted by $\left[{ }^{\alpha} a,{ }^{\alpha} c,{ }^{\alpha} b\right]$, shown in Figure 1, whose membership function is:

$$
\mu(x)=\left\{\begin{array}{cccc}
0 & , & \text { if } & x \leq{ }^{\alpha} a \text { and } x \geq{ }^{\alpha} b \\
\frac{x-{ }^{\alpha} a}{{ }^{\alpha} c-{ }^{\alpha} a} & , & \text { if } & { }^{\alpha} a<x<{ }^{\alpha} c \\
1, & , \text { if } & x={ }^{\alpha} c \\
\frac{{ }^{\alpha} b-x}{{ }^{\alpha} b-{ }^{\alpha} c} & , \text { if } & { }^{\alpha} c<x<{ }^{\alpha} b
\end{array}\right.
$$

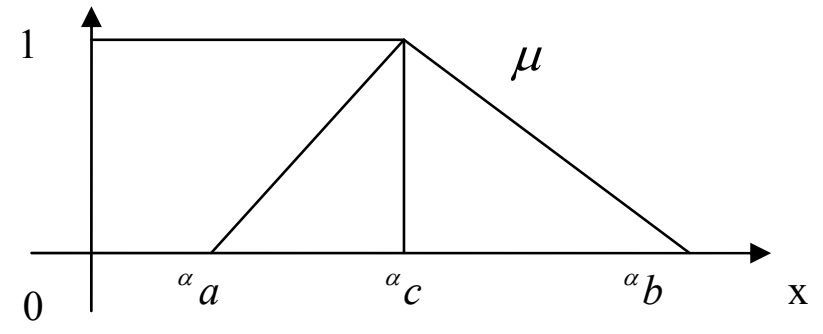

Figure 1: Fuzzy Number.

Here it is noted that, if $\mu$ is a continuous fuzzy number which is increasing, or $\mu$ is a continuous fuzzy number which is decreasing then ${ }^{\alpha} b={ }^{\alpha} c$, or ${ }^{\alpha} a={ }^{\alpha} c$, respectively, shown in Figure - 2.
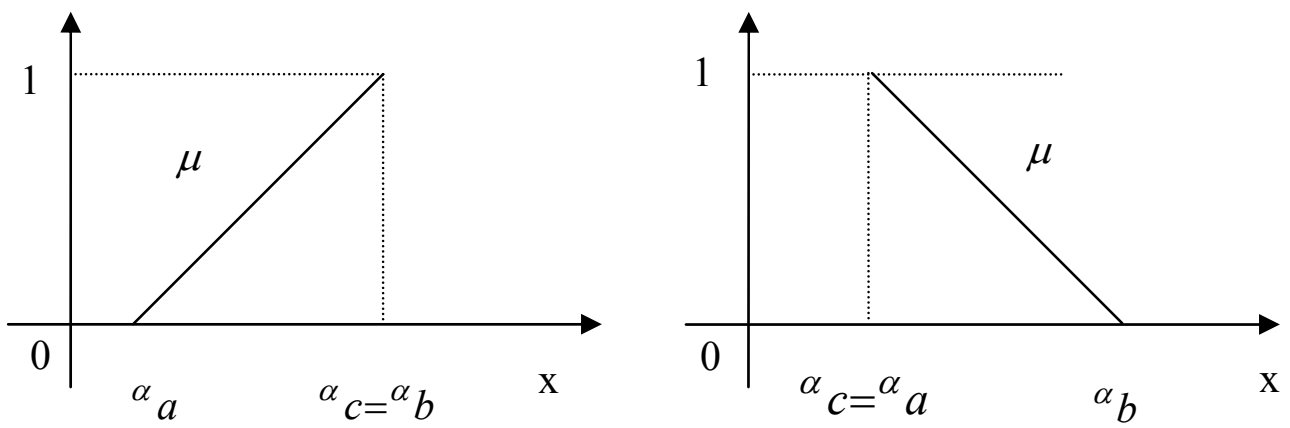

Figure 2 : Increasing and decreasing part of a Fuzzy Number.

The definitions of MAX and MIN which are the lattice operations to be used in the ordering of fuzzy numbers are as follows [6] : 
Definition 2.3: Let $\mu_{1}, \mu_{2} \in \phi v(\mathrm{P})$. Then, for all $x_{1}, x_{2}, z \in \mathrm{P}$,

$$
\begin{aligned}
& \operatorname{MAX}\left(\mu_{1}, \mu_{2}\right)(\mathrm{z})=\sup _{z=\max \left(x_{1}, x_{2}\right)} \min \left[\mu_{1}\left(x_{1}\right), \mu_{2}\left(x_{2}\right)\right] \\
& \operatorname{MIN}\left(\mu_{1}, \mu_{2}\right)(\mathrm{z})=\sup _{z=\min \left(x_{1}, x_{2}\right)} \min \left[\left[\mu_{1}\left(x_{1}\right), \mu_{2}\left(x_{2}\right)\right] .\right.
\end{aligned}
$$

In general, they are defined for any finite number of fuzzy numbers $\mu_{i}$, and for $x_{i} \in \mathrm{P}$, $i=1,2, \cdots, n$ as follows:

$$
\begin{aligned}
& \operatorname{MAX}\left(\mu_{1}, \mu_{2}, \cdots, \mu_{n}\right)(\mathrm{z})=\sup _{z=\max \left(x_{1}, x_{2}, \cdots, x_{n}\right)} \min \left[\mu_{1}\left(x_{1}\right), \mu_{2}\left(x_{2}\right), \cdots, \mu_{n}\left(x_{n}\right)\right] \\
& \operatorname{MIN}\left(\mu_{1}, \mu_{2}, \cdots, \mu_{n}\right)(\mathrm{z})=\sup _{z=\min \left(x_{1}, x_{2}, \cdots, x_{n}\right)} \min \left[\left[\mu_{1}\left(x_{1}\right), \mu_{2}\left(x_{2}\right), \cdots, \mu_{n}\left(x_{n}\right)\right] .\right.
\end{aligned}
$$

Recently, Hong and Kim [5] proposed the following method to implement the operations MAX and MIN for fuzzy numbers using $\alpha$-cut representation.

Theorem 2.2: Let $A_{i}, i=1, \cdots, n$, be fuzzy numbers with $\left[A_{i}\right]^{\alpha}=\left[a_{i}^{1}, a_{i}^{2}\right]$. Then the operation MIN and MAX can be implemented as

$$
\begin{aligned}
& {\left[\operatorname{MIN}\left(A_{1}, \cdots, A_{n}\right)\right]^{\alpha}=\left[\min _{1 \leq i \leq n} a_{i}^{1}(\alpha), \min _{1 \leq i \leq n} a_{i}^{2}(\alpha)\right],} \\
& {\left[\operatorname{MAX}\left(A_{1}, \cdots, A_{n}\right)\right]^{\alpha}=\left[\max _{1 \leq i \leq n} a_{i}^{1}(\alpha), \max _{1 \leq i \leq n} a_{i}^{2}(\alpha)\right] .}
\end{aligned}
$$

\section{The New Approach of $\alpha$-cut Presentation of MAX and MIN}

Here we proposed the method to implement the $\alpha$-cut presentation of the operations MAX and MIN for fuzzy numbers using Definition 2.3. It is noted that if $\mu_{1}$ and $\mu_{2}$ are fuzzy numbers, then $\operatorname{MAX}\left(\mu_{1}, \mu_{2}\right)$ and $\operatorname{MIN}\left(\mu_{1}, \mu_{2}\right)$ are also fuzzy numbers.

Theorem 3.1: Let $\mu_{i}, i=1,2, \cdots, n$, be fuzzy numbers with their $\alpha$-cuts ${ }^{\alpha} \mu_{i}=\left[{ }^{\alpha} a_{i},{ }^{\alpha} c_{i},{ }^{\alpha} b_{i}\right]$. Then the $\alpha$-cuts of $\operatorname{MAX}\left(\mu_{1}, \cdots, \mu_{n}\right)$ and $\operatorname{MIN}\left(\mu_{1}, \cdots, \mu_{n}\right)$ are 


$$
\begin{aligned}
& \text { (i) }{ }^{\alpha}\left[\operatorname{MAX}\left(\mu_{1}, \cdots, \mu_{n}\right)\right]=\left[\max _{1 \leq i \leq n} \alpha_{a_{i}, \max }{ }_{1 \leq i \leq n}^{\alpha} c_{i}, \max _{1 \leq i \leq n} \alpha b_{i}\right] \text {; } \\
& \text { (ii) }{ }^{\alpha}\left[\operatorname{MIN}\left(\mu_{1}, \cdots, \mu_{n}\right)\right]=\left[\min _{1 \leq i \leq n} \alpha a_{i}, \min _{1 \leq i \leq n} \alpha c_{i}, \min _{1 \leq i \leq n} \alpha b_{i}\right] .
\end{aligned}
$$

Proof: (i) Consider any $z \in \mathrm{P}$. Then for $x_{i} \in \mathrm{P}, i=1, \cdots, n$,

$z \in^{\alpha}\left[\operatorname{MAX}\left(\mu_{1}, \cdots, \mu_{n}\right)\right]$

$\Leftrightarrow\left[\operatorname{MAX}\left(\mu_{1}, \cdots, \mu_{n}\right)\right](z) \geq \alpha$

$\Leftrightarrow$ for some $i$,

$\sup _{x_{i}=z, x_{j} \leq z, j \neq i} \min \left(\mu_{1}\left(x_{1}\right), \cdots, \mu_{n-1}\left(x_{n-1}\right), \mu_{i}(z), \cdots, \mu_{n}\left(x_{n}\right) \geq \alpha\right.$

$\Leftrightarrow$ for some $i$,

$\mu_{i}(z) \geq \alpha$ and for some $j \neq i, \exists y_{i}={ }^{\alpha} a_{j}$

such that $\mu_{i}\left(y_{i}\right)=\alpha, y_{i} \leq y_{j} \leq z$ and $\mu_{j}\left(y_{j}\right) \geq \alpha$

$\Leftrightarrow$ for some $i$,

$z \in{ }^{\alpha} \mu_{i}=\left[{ }^{\alpha} a_{i},{ }^{\alpha} c_{i},{ }^{\alpha} b_{i}\right]$,

where $z \in\left[{ }^{\alpha} a_{i},{ }^{\alpha} c_{i}\right]$ or $z \in\left[{ }^{\alpha} c_{i},{ }^{\alpha} b_{i}\right]$ and for some $j \neq i, z \geq{ }^{\alpha} a_{j}$

$\Leftrightarrow z \in \bigcup_{i=1}^{n} \alpha \mu_{i}=\bigcup_{i=1}^{n}\left[\alpha a_{i},{ }^{\alpha} c_{i},{ }^{\alpha} b_{i}\right]$, and $z \geq \max _{1 \leq j \leq n}\left({ }^{\alpha} a_{j}\right)$

$\Leftrightarrow z \in\left[\max _{1 \leq i \leq n} \alpha^{\alpha} a_{i}, \max _{1 \leq i \leq n} \alpha^{\alpha} c_{i, \max _{1 \leq i \leq n}} \alpha^{\alpha} b_{i}\right]$

Hence, ${ }^{\alpha}\left[\operatorname{MAX}\left(\mu_{1}, \cdots, \mu_{n}\right)\right]=\left[\max _{1 \leq i \leq n}{ }^{\alpha} a_{i}, \max _{1 \leq i \leq n}{ }^{\alpha} c_{i}, \max _{1 \leq i \leq n}{ }^{\alpha} b_{i}\right]$.

(ii) Consider any $z \in \mathrm{P}$. Then for $x_{i} \in \mathrm{P}, i=1, \cdots, n$,

$$
\begin{aligned}
& z \in^{\alpha}\left[\operatorname{MIN}\left(\mu_{1}, \cdots, \mu_{n}\right)\right] \\
& \Leftrightarrow\left[\operatorname{MIN}\left(\mu_{1}, \cdots, \mu_{n}\right)\right](z) \geq \alpha
\end{aligned}
$$


$\Leftrightarrow$ for some $i$,

$$
\begin{aligned}
& \sup _{x_{i}=z, x_{j} \geq z, j \neq i} \min \left(\mu_{1}\left(x_{1}\right), \cdots, \mu_{n-1}\left(x_{n-1}\right), \mu_{i}(z), \cdots, \mu_{n}\left(x_{n}\right) \geq \alpha\right. \\
& \Leftrightarrow \text { for some } i,
\end{aligned}
$$$$
\mu_{i}(z) \geq \alpha \text { and for all } j \neq i, \exists y_{i}={ }^{\alpha} b_{j}
$$$$
\text { such that } \mu_{i}\left(y_{i}\right)=\alpha, z \leq y_{j} \leq y_{i} \text { and } \mu_{j}\left(y_{j}\right) \geq \alpha
$$

$\Leftrightarrow$ for some $i$,

$z \in{ }^{\alpha} \mu_{i}=\left[{ }^{\alpha} a_{i},{ }^{\alpha} c_{i},{ }^{\alpha} b_{i}\right]$,

where $z \in\left[{ }^{\alpha} a_{i},{ }^{\alpha} c_{i}\right]$ or $z \in\left[{ }^{\alpha} c_{i},{ }^{\alpha} b_{i}\right]$ and for all $j \neq i, z \leq{ }^{\alpha} b_{j}$

$\Leftrightarrow z \in \bigcup_{i=1}^{n}{ }^{\alpha} \mu_{i}=\bigcup_{i=1}^{n}\left[\alpha a_{i},{ }^{\alpha} c_{i},{ }^{\alpha} b_{i}\right]$, and $z \leq \min _{1 \leq j \leq n}\left({ }^{\alpha} b_{j}\right)$

$\Leftrightarrow z \in\left[\min _{1 \leq i \leq n}{ }^{\alpha} a_{i}, \min _{1 \leq i \leq n}{ }^{\alpha} c_{i}, \min _{1 \leq i \leq n} \alpha b_{i}\right]$.

Hence, ${ }^{\alpha}\left[\operatorname{MIN}\left(\mu_{1}, \cdots, \mu_{n}\right)\right]=\left[\min _{1 \leq i \leq n} \alpha_{a_{i}, \min _{1 \leq i \leq n}}{ }^{\alpha} c_{i}, \min _{1 \leq i \leq n}{ }^{\alpha} b_{i}\right]$.

\section{The Method to Compute the Membership Functions and Results}

Let $\mu_{i}, i=1,2, \cdots, n$, be fuzzy numbers. Then every fuzzy number has a bounded increasing function on $[0,1]$ and a bounded decreasing function on $[0,1]$, by Theorem 2.2. Suppose that all or some of them intersect at some points, or none of them intersects. That is, it is supposed that either $\bigwedge_{i} \mu_{i} \neq 0$ for all $i$, or $\bigwedge_{i} \mu_{i} \neq 0$ for some $i$, or $\bigwedge_{i} \mu_{i}=0$ for all $i$. We now focus on a method to compute the membership functions of maximum and minimum, which are denoted by $\operatorname{MAX}\left(\mu_{i}\right)$ and $\operatorname{MIN}\left(\mu_{i}\right)$, respectively, of the fuzzy numbers and discuss the solution of a problem using the method.

Method 4.1: Let $\left(x_{k}, \eta_{i}\left(x_{k}\right)\right)$ and $\left(y_{l}, v_{i}\left(y_{l}\right)\right), k, l \in I N$, be intersecting points of bounded increasing functions $\eta_{i}$ and bounded decreasing functions $v_{i}$ of the fuzzy numbers $\mu_{i}, i=1, \cdots, \quad n, \quad$ in the intervals $\left[\max \left({ }^{\alpha-1} a_{i}\right), \max \left({ }^{\alpha_{k}} c_{i}\right)\right]$, $\left[\max \left({ }^{\beta_{l}} c_{i}\right), \max \left({ }^{\beta_{l-1}} b_{i}\right)\right]$ such that $\alpha_{k}=\eta_{i}\left(x_{k}\right), \beta_{l}=v_{i}\left(y_{l}\right)$, respectively, where 
$\max \left({ }^{\alpha_{k-1}} a_{i}\right) \leq x_{1} \leq \cdots \leq x_{k} \leq \max \left({ }^{\beta_{0}} c_{i}\right), \quad \max \left({ }^{\beta_{0}} a_{i}\right) \leq y_{1} \leq \cdots \leq y_{l} \leq \max \left({ }^{\alpha_{0}} c_{i}\right)$ and $0=\alpha_{0} \leq \alpha_{1} \leq \cdots \leq \alpha_{k} \leq 1, \quad 1=\beta_{0} \geq \beta_{1} \geq \cdots \geq \beta_{l} \geq 0$. Let, $\quad \max \left({ }^{\alpha_{0}} a_{i}\right)=x_{0}$, and $\max \left({ }^{\beta_{0}} c_{i}\right)=x_{\max (\mathrm{k})}=y_{0}$. Then

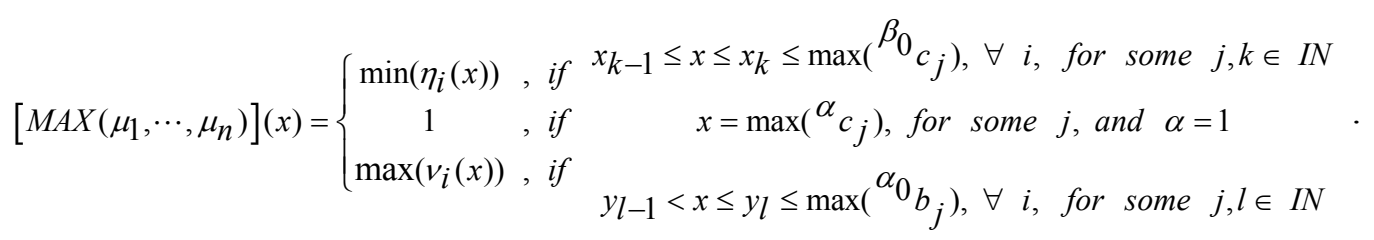

Method 4.2: Let $\left(x_{k}, \eta_{i}\left(x_{k}\right)\right)$ and $\left(y_{l}, v_{i}\left(y_{l}\right)\right), k, l \in I N$, be intersecting points of bounded increasing functions $\eta_{i}$ and bounded decreasing functions $v_{i}$ of the fuzzy numbers $\mu_{i}, i=1, \cdots, n, \quad$ in the intervals $\quad\left[\min \left({ }^{\alpha_{k-1}} a_{i}\right), \min \left({ }^{\alpha_{k}} c_{i}\right)\right]$, $\left[\min \left({ }^{\beta_{l}} c_{i}\right), \min \left({ }^{\beta_{l-1}} b_{i}\right)\right]$ such that $\alpha_{k}=\eta_{i}\left(x_{k}\right), \beta_{l}=v_{i}\left(y_{l}\right)$, respectively, where $\min \left({ }^{\alpha_{k-1}} a_{i}\right) \leq x_{1} \leq \cdots \leq x_{k} \leq \min \left({ }^{\beta_{0}} c_{i}\right), \quad \min \left({ }^{\beta_{0}} c_{i}\right) \leq y_{1} \leq \cdots \leq y_{l} \leq \min \left({ }^{\alpha_{0}} b_{i}\right) \quad$ and $0=\alpha_{0} \leq \alpha_{1} \leq \cdots \leq \alpha_{k} \leq 1, \quad 1=\beta_{0} \geq \beta_{1} \geq \cdots \geq \beta_{l} \geq 0$. Let, $\quad \min \left({ }^{\alpha_{0}} a_{i}\right)=x_{0}$, and $\min \left({ }^{\beta_{0}} c_{i}\right)=y_{0}$. Then

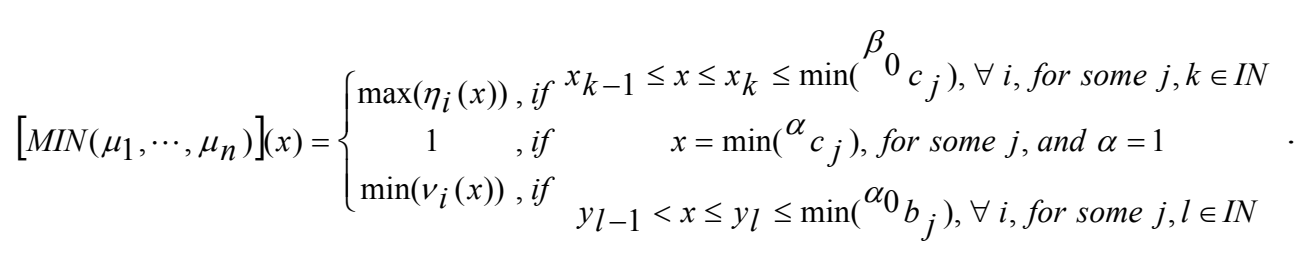

Example: Consider the fuzzy numbers $\mu_{i}, i=1,2,3$, defined by

$$
\mu_{1}(x)=\left\{\begin{array}{c}
\frac{x+2}{2}, \text { if }-2 \leq x \leq 0 \\
\frac{6-x}{6}, \text { if } 0 \leq x \leq 6 \\
0, \text { Otherwise }
\end{array}\right.
$$




$$
\mu_{2}(x)=\left\{\begin{array}{c}
\frac{x+4}{7}, \text { if }-4 \leq x \leq 3 \\
\frac{5-x}{2}, \text { if } 3 \leq x \leq 5 \\
0, \text { Otherwise }
\end{array}\right.
$$

$$
\mu_{3}(x)=\left\{\begin{array}{c}
\frac{x+1}{3}, \text { if }-1 \leq x \leq 2 \\
\frac{8-x}{6}, \text { if } 2 \leq x \leq 8 . \\
0, \text { Otherwise }
\end{array} .\right.
$$

The graphs of $\mu_{1}, \mu_{2}$, and $\mu_{3}$ are shown in Figure 3. Now we compute the membership functions of the fuzzy numbers $\operatorname{MAX}\left(\mu_{1}, \mu_{2}, \mu_{3}\right)$ and $\operatorname{MIN}\left(\mu_{1}, \mu_{2}, \mu_{3}\right)$.

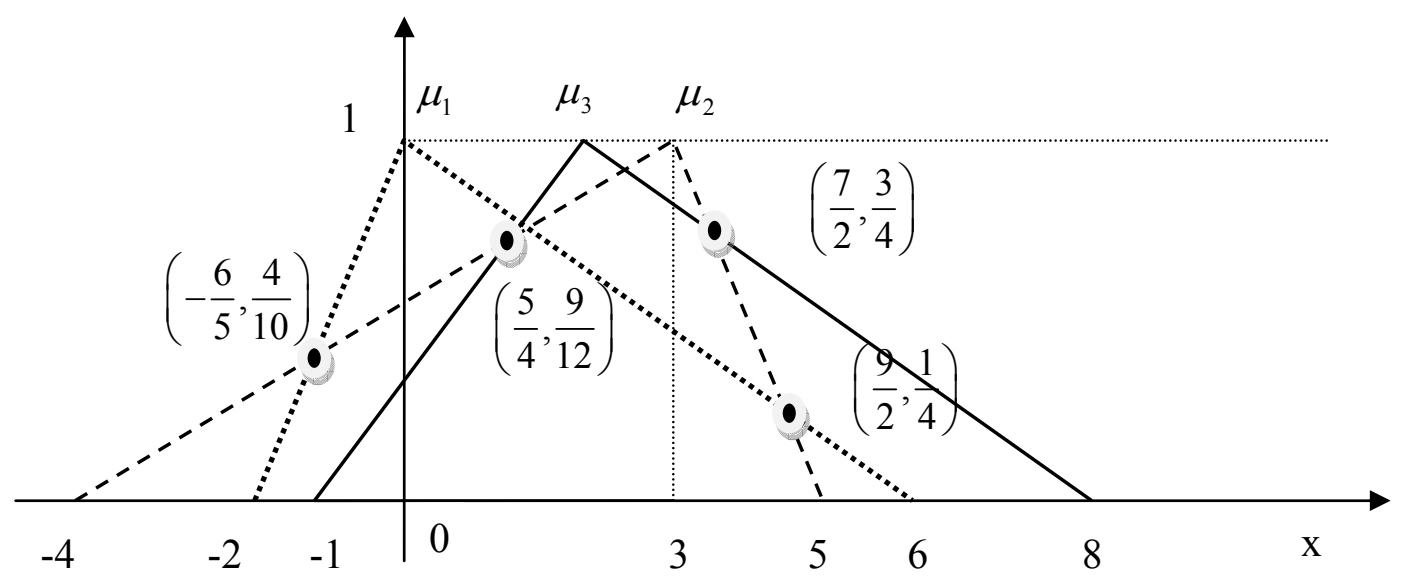

Figure 3: Graphical representations of Fuzzy Numbers $\mu_{1}, \mu_{2}$ and $\mu_{3}$.

\section{For MAX operation:}

There are intersecting points $\left(\frac{5}{4}, \frac{9}{12}\right)$ in the interval $\left[\max \left({ }^{\alpha} a_{i}\right)=-1, \max \left({ }^{\beta_{0}} c_{i}\right)=3\right]$ and $\left(\frac{7}{2}, \frac{3}{4}\right), \quad\left(\frac{9}{2}, \frac{1}{4}\right) \quad$ in the interval $\left[\max \left({ }^{\beta_{0}} c_{i}\right)=3, \max \left({ }^{\alpha_{0}} b_{i}\right)=8\right]$. Here, $x_{0}=-1, x_{1}=\frac{5}{4}$, and $y_{0}=3, y_{1}=\frac{7}{2}, y_{2}=\frac{9}{2}$. Therefore, using Theorem 4.1, 


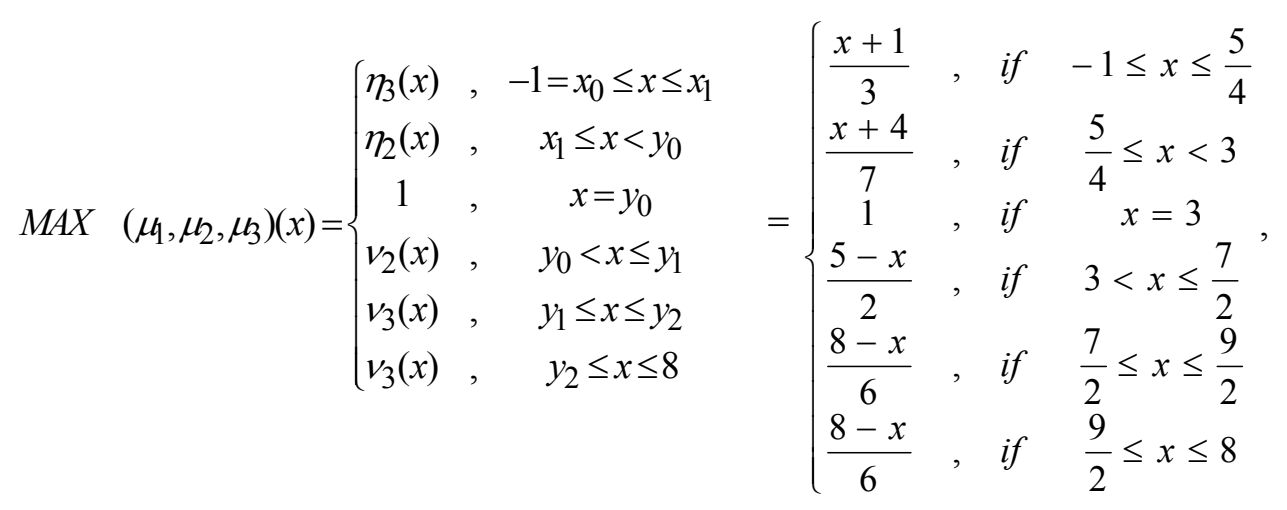

which is equivalent to

$$
\operatorname{MAX}\left(\mu_{1}, \mu_{2}, \mu_{3}\right)(\mathrm{x})=\left\{\begin{array}{cccc}
\frac{x+1}{3} & , & \text { if } & -1 \leq x \leq \frac{5}{4} \\
\frac{x+4}{7} & , & \text { if } & \frac{5}{4} \leq x<3 \\
1, & , & \text { if } & x=3 \\
\frac{5-x}{2} & , & \text { if } & 3<x \leq \frac{7}{2} \\
\frac{8-x}{6} & , & \text { if } & \frac{7}{2} \leq x \leq 8
\end{array} .\right.
$$

\section{For MIN operation:}

There are intersecting points $\left(-\frac{6}{5}, \frac{4}{10}\right)$ in the interval $\left[\min \left({ }^{\alpha} a_{i}\right)=-4, \min \left({ }^{\beta_{0}} c_{i}\right)=0\right]$ and $\left(\frac{9}{2}, \frac{1}{4}\right)$ in the interval $\left[\min \left({ }^{\beta_{0}} c_{i}\right)=0, \min \left({ }^{\alpha_{0}} b_{i}\right)=5\right]$. Similarly, using Theorem 4.2,

$$
\operatorname{MIN}\left(\mu_{1}, \mu_{2}, \mu_{3}\right)(\mathrm{x})=\left\{\begin{array}{cccc}
\frac{x+4}{7}, & \text { if } & -4 \leq x \leq-\frac{6}{5} \\
\frac{x+2}{2}, & \text { if } & -\frac{6}{5} \leq x<0 \\
1 & , & \text { if } & x=0 \\
\frac{6-x}{6}, & \text { if } & 0<x \leq \frac{9}{2} \\
\frac{5-x}{2}, & \text { if } & \frac{9}{2} \leq x \leq 5
\end{array} .\right.
$$


The graphs of $\operatorname{MAX}\left(\mu_{1}, \mu_{2}, \mu_{3}\right)$ and $\operatorname{MIN}\left(\mu_{1}, \mu_{2}, \mu_{3}\right)$ are shown in Figure 4.

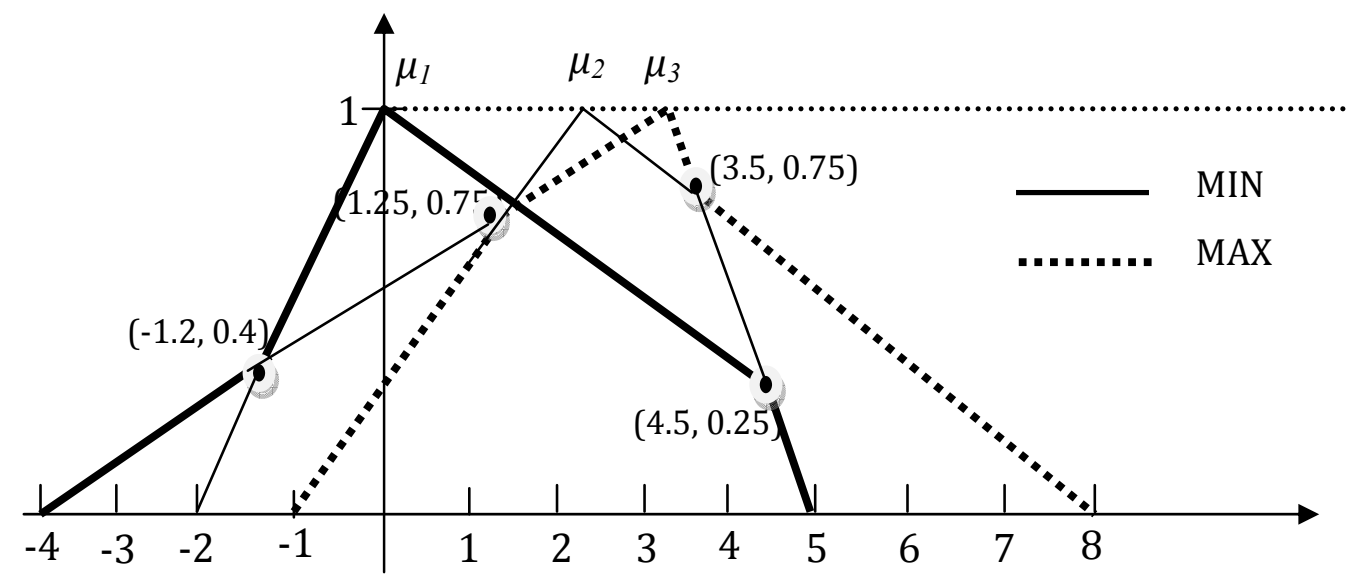

Figure-4 : The graphs of $\operatorname{MAX}\left(\mu_{1}, \mu_{2}, \mu_{3}\right)$ and $\operatorname{MIN}\left(\mu_{1}, \mu_{2}, \mu_{3}\right)$.

Remark: We can generalize the Theorem 4.1 and Theorem 4.2 for supremum and infimum, which are denoted by SUP $\left(\mu_{i}\right)$ and INF $\left(\mu_{i}\right)$ respectively, of any numbers of fuzzy numbers. To perform these we use 'sup' and 'inf' instead of 'max' and 'min', respectively.

\section{Conclusion}

In this paper, we have introduced a method which is capable of computing the membership functions $\operatorname{MAX}\left(\mu_{i}\right)$ and $\operatorname{MIN}\left(\mu_{i}\right)$ of the maximum and the minimum of any finite numbers of fuzzy numbers $\mu_{i}, i=1,2, \cdots, n$ directly, but HONG and KIM's [5] method does not work in such a way. We threw also the concept of SUP and INF operation of the fuzzy numbers as a generalization of maximum (MAX) and minimum (MIN) of them, respectively.

\section{REFERENCES}

1. Chiu, C. H., Wang, W. J., A simple computation of MIN and MAX operations for fuzzy numbers, Fuzzy Sets and Systems, 126, (2002), p. 273-276.

2. Dubois, D., Prade, H., Operations on fuzzy numbers, Internet. J. Systems Science, 9(6), (1978),p. 613626. 
3. Giachetti, R. E., Young, R. E., A parametric representation of fuzzy numbers and their arithmetic operators, Fuzzy Sets and Systems, 91, (1997), p. 185-202.

4. Goetschel, R., and Voxman, W., Elementary fuzzy calculus, Fuzzy Sets and Systems, 18, (1986), p. 3143.

5. HONG, D. H, and KIM, K. T., An easy computation of MIN and MAX operations for fuzzy numbers, J. Appl. Math. \& Computing, Vol. 21, (2006), No. 1-2, p. 555-561.

6. Klir, G. J., Yua, B., Fuzzy Sets and Fuzzy Logic Theory and Applications, Prentice-Hall of India Private Limited, New Delhi, (1995), p. 95-114.

7. Zadeh, L. A., Fuzzy Sets, Information and Control, 8(3), (1965), p. 338-353. 